\title{
Mitochondrial myopathy and sideroblastic anemia
}

INSERM

\section{Source}

INSERM. (1999). Orphanet: an online rare disease and orphan drug data base.

Mitochondrial myopathy and sideroblastic anemia. ORPHA:2598

Mitochondrial myopathy and sideroblastic anemia belongs to the heterogeneous family

of metabolic myopathies. It is characterised by progressive exercise intolerance

manifesting in childhood, onset of sideroblastic anaemia around adolescence, lactic acidaemia, and mitochondrial myopathy. 